\title{
EVENTRATION OF THE DIAPHRAGM, A RISK FACTOR FOR RECURRENT RESPIRATORY TRACT INFECTION - A CASE REPORT
}

\author{
Mihaela Ciornei ${ }^{1}$, Elena Hanganu${ }^{1}$, Diana Ecaterina Popa ${ }^{1}$, T. Stanciu ${ }^{2}$, \\ C. Gheonea ${ }^{3}$, B. Savu ${ }^{1}$ \\ ${ }^{1}$ Pediatric Surgery Department, \\ „,Gr. T. Popa “, University of Medicine and Pharmacy, Iasi \\ ${ }^{2}$ Neonatology Department, ,, Gr. T. Popa “ University of Medicine and Pharmacy, Iasi \\ ${ }^{3}$ Pediatric Department, University of Medicine and Pharmacy, Craiova
}

\begin{abstract}
Eventration of the diaphragm, congenital or acquired, represents a rare condition (1 to 10,000 live births), in which the dome of the diaphragm is abnormally elevated, due to an excessive amount of fibro-elastic diaphragmatic tissue. The child may be asymptomatic or present with respiratory symptoms as aggressive as life threatening respiratory distress. Recurrent pulmonary infections are the commonest presenting complaints. We present the case of a 3 years old boy with recurrent chest infections and apparent "dextrocardia", both caused by a left eventration of the diaphragm, successfully treated by plication.
\end{abstract}

Keywords: diaphragm eventration, respiratory infections, plication

\section{INTRODUCTION}

Eventration of the diaphragm, congenital or acquired, is defined as the permanent elevation of the diaphragm without an anatomic defect of continuity. It is a rare condition, 1 in 10,000 live births. The diaphragm's muscular tissue is replaced by a fibroelastic structure, affecting the attachments to the sternum, ribs, and dorsolumbar spine, with normally sealed orifices and no interruption of the pleural or peritoneal layers (1).

Symptoms in the eventration of the diaphragm may present as unspecific or minor, causing diagnostic misinterpretation in some cases. In asymptomatic patients, diagnosis is achieved incidentally, when exploration for other problems is performed. The most evident symptoms in diaphragmatic eventration are caused by the ascension of bowel loops into the thorax that compress the inferior pulmonary lobe, leading to cyanosis, respiratory failure and cardio-vascular dysfunction. Anorexia, em- esis and vomiting may also be encountered in diaphragmatic eventration patients. These symptoms may be triggered by several causes: 1) A ventilatory impairment of the ipsilateral lung, through its direct compression. 2) A pneumonitis caused by atelectasis. 3) Inefficient ventilation of the contra lateral lung, secondary to the paradoxical movements of the diaphragm. 4) The "pendelluft" effect, between the affected and the healthy lung, during ventilation(2).

Due to the increased mobility of the mediastinum in children, the abnormal movements of the diaphragm will cause a mediastinal and cardiac shifting towards the contra lateral hemi thorax. In patients with diaphragmatic eventration, the vital capacity, as well as the total pulmonary capacity is diminished by $20-30 \%$. This leads to a narrowing of the thorax, recurrent respiratory infections and mild colds, with or without expectoration. In the neonatal period, a clinical picture of respiratory 
failure, cyanosis, tachycardia and arrhythmia may be encountered (2).

Imaging diagnosis of diaphragmatic eventration is most frequently achieved on chest radiographs, in antero-posterior and lateral incidence that show the bowel loop ascension. On chest fluoroscopy, the lateral view is essential to the evaluation, especially for the lesion on the right side, as it will show the paradoxical diaphragmatic movements. These movements may also be explored through ultrasound examination. Such movements may impair gas exchange and pulmonary perfusion. For a more precise diagnosis of the condition, the CT scan and / or MRI may be used. A diaphragmatic eventration on the right side may be difficult to differentiate from a congenital diaphragmatic hernia $(\mathrm{CDH})$, because of the presence of the liver ascension; in such cases, a thoracoscopy may be required (2).

Indications for surgery in diaphragmatic eventration include: 1) increased respiratory frequency without improvement under a conservative treatment; 2) recurring ipsilateral pneumonias; 3) one life-threatening pneumonia; 4) inability to wean from mechanical ventilation; 5) respiratory distress related to paradoxical motion of the diaphragm. The diaphragm is plicated through an abdominal or thoracic approach, by either open or minimally invasive techniques. The small forms of eventration are amenable to a conservative treatment. The first report of a surgical repair of diaphragmatic eventration in an adult was published by Morrison in 1923 (3). In 1964 Bisgard performed the procedure in a child for the first time (2).

\section{CASE PRESENTATION}

G.M. a 3-year-old boy, coming from a rural area, had several admissions in territorial pediatric services for recurrent respiratory infection episodes. During the last clinical examination, the pediatrician had noticed a minor deformity of the left thorax with a right shift of cardiac auscultation and a diminished respiratory murmur on the left pulmonary area; these, associated to the many respiratory infections in the past, raised the suspicion of a congenital diaphragmatic hernia, so the child was addressed to the pediatric surgery service, a week after the respiratory infection remission. The child had been born through a full term normal delivery of a monitored pregnancy; birth weight was $3,000 \mathrm{~g}$; there was no history of maternal fever or erythematous rashes during pregnancy; fetal ultrasound check-ups had shown no abnormal details. After birth, the mother declared there had been numerous episodes of respiratory problems that were treated without hospital admissions.

On physical examination performed by the authors, the following features were noticed: mild body hypotrophy $(-2,25 \mathrm{DS})$; microcephaly $(-2,39$ DS); no signs of respiratory distress; skin pallor; mild dehydration; no facial dysmorphism; discreet asymmetric distended chest, with decreased movements on left infra-mammary, infra-axillary, and infrascapular area; respiratory rate of 33/minute, diminished respiratory sounds on the left infra-axillary and infrascapular areas; cardiac sounds auscultation shifted to the right side (Fig.1).

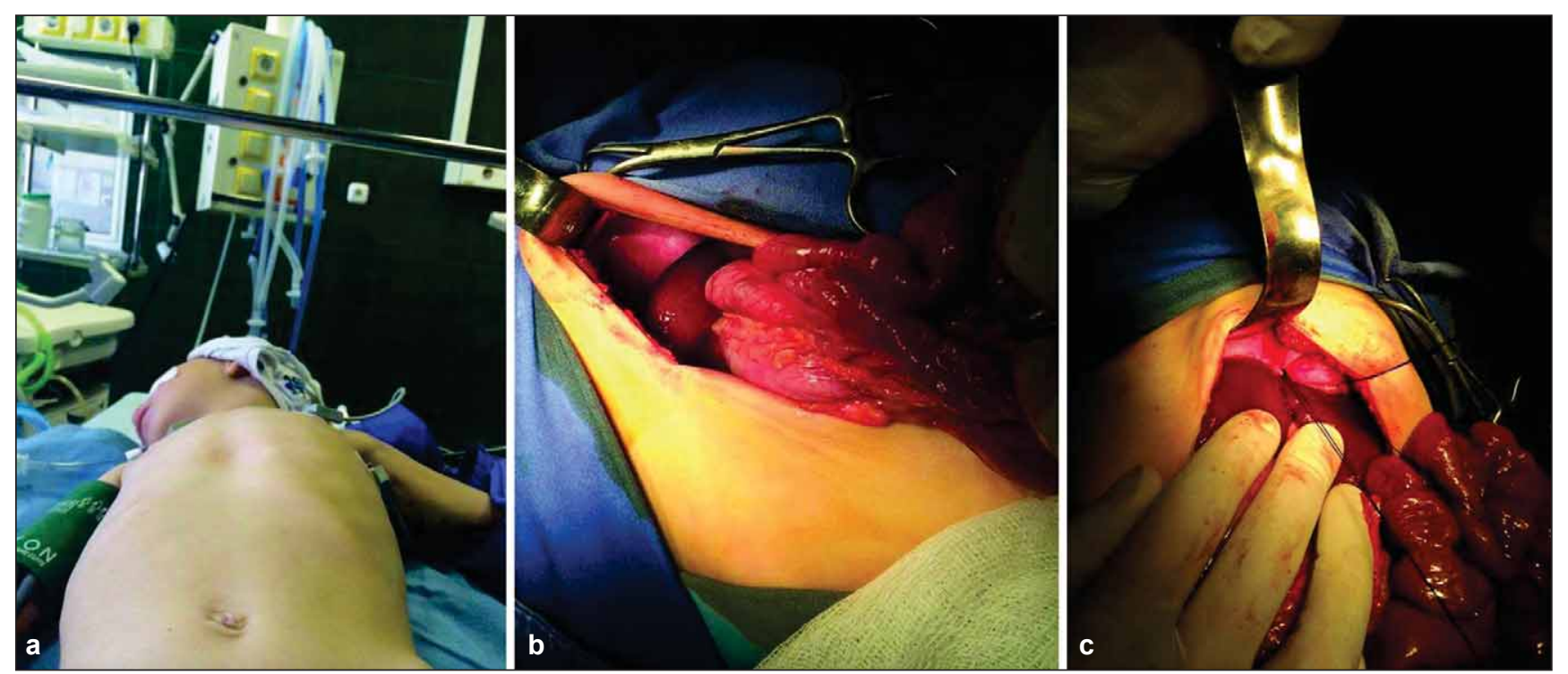

FIGURE 1. a. Clinical aspect of the thorax b. Intraoperatory aspect: abdominal viscera in the thorax, eventration of the diaphragm c. Intraoperatory aspect after plicature 


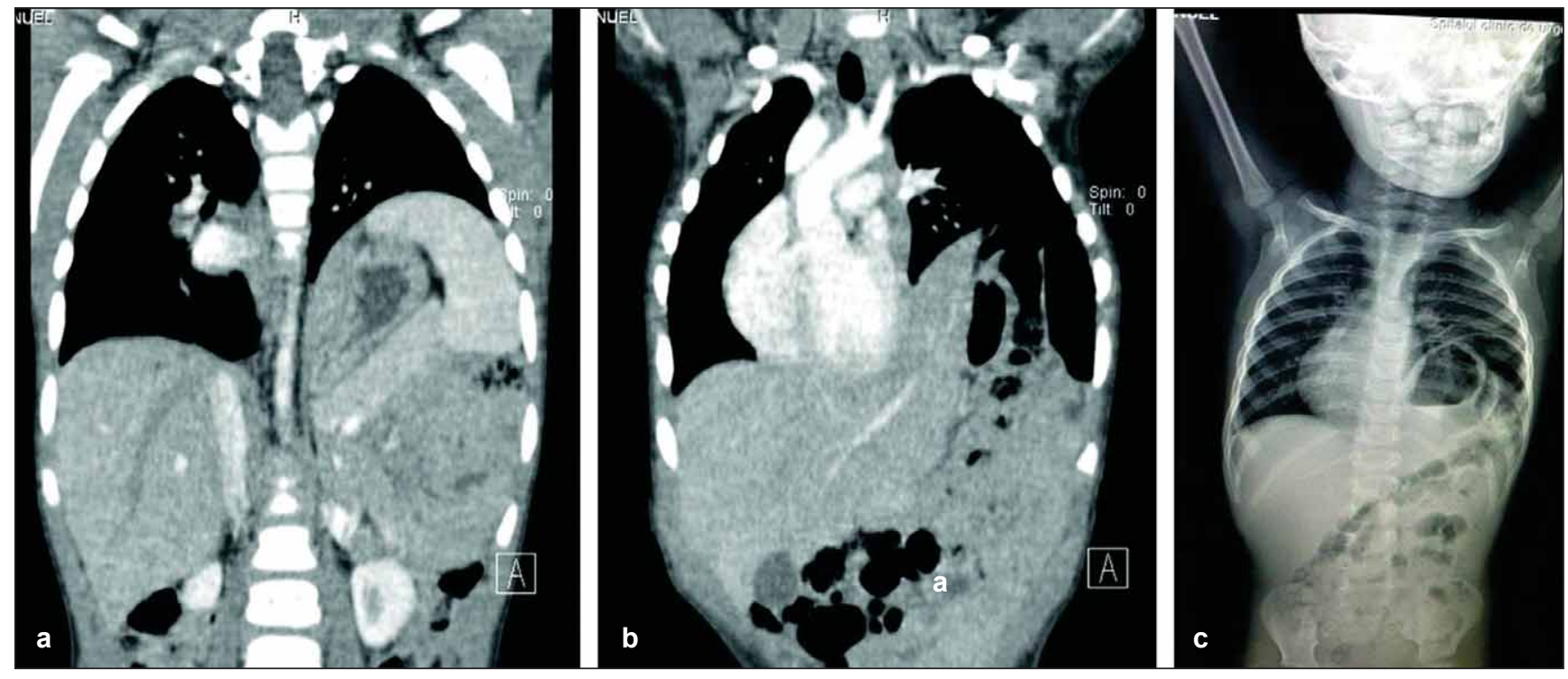

FIGURE 2. a. CT: displacement of the spleen and pancreas into thorax; b. CT: mediastinal displacement; c. Rx: bowel in to the thorax

Plain antero-posterior chest radiograph demonstrated the presence of bowel loops and of the gastric air bubble in the left hemi thorax, shifting of the cardiac silhouette to the right thorax, bilateral normal aeration of the pulmonary parenchyma (Fig .2).

Echocardiography, abdominal and renal ultrasonography showed no other associated anomalies. The thoracic CT scan suggested the presence of a left congenital diaphragmatic hernia (Fig. 2). Other contrast studies were not considered necessary. Hematological and biochemical workup showed a normal picture.

The operative approach was decided. Under general anesthesia, a left sub costal laparotomy was performed, leading to the finding of an abnormal elevation of the left diaphragmatic dome, with paradoxical diaphragmatic movements, but no anatomic defect. The left lobe of the liver, the spleen, and several bowel loops where ascended into the chest, with an incomplete intestinal malrotation. Surgical plication of the left diaphragmatic dome was performed (Fig. 2). The postoperative course was uneventful. Chest films performed after plication showed the left diaphragm maintaining a proper position, with no ascended bowel loop. The child was discharged 7 days after surgery with good digestive tolerance and no respiratory symptoms. Recommendations on discharge included dietary adjustments for malnutrition recovery. The patient is currently on regular follow up, showing no particular symptoms.

\section{DISCUSSIONS}

The eventration of the diaphragm is caused by the atrophy and progressive distension of the mus- cular dome. The diaphragmatic attachments to the sternum, ribs, and dorsolumbar spine are abnormally shaped. The aortic and esophageal orifices are normal and the pleura and peritoneum are intact. During the neonatal period, the acute respiratory failure is the most frequently encountered syndrome, while older children and adults might be asymptomatic or presenting with recurrent respiratory infections, dyspnoea and thoracic pain. A difficult differential diagnosis in some cases is involving the possible presence of a $\mathrm{CDH}$ with herniary sac. The embryologic etiology is due to an abnormal migration of myoblasts from the upper cervical somites into two of the four embryological structures that contribute to diaphragm development such as septum transversum (beginning at 4 weeks of gestation) and pleuroperitoneal membrane (at 8 to 12 weeks of gestation) (2). As DeVries et al clearly showed, the phrenic nerve injury inducing chronic diaphragmatic paralysis is the main cause of acquired eventration of the diaphragm (4), when the diaphragmatic muscle is present in its normal distribution, but is inactive and attenuated, and the long-lasting diaphragm paralysis may lead to cor pulmonale (2). Paralysis of the hemi diaphragm causes atelectasis and ventilation-perfusion mismatch, preferential redistribution of pulmonary blood flow to the contra lateral lung, shift of the mobile mediastinum to the contra lateral side and paradoxical movements of the affected hemidiaphragm (5). Diaphragmatic plication is the standard treatment to treat diaphragmatic eventration. Though it was described since 1923, there have been only a limited number of reports published, 
most of these presenting small series with short follow-ups $(6,7)$, both in adults and children.

Simansky et al. proposed in 2002 as indications for surgery in affected children the failure to wean from ventilatory support symptomatic dyspnea (8). Till recently an open approach through thoracotomy or laparotomy was the standard, but Freeman et al. reported a series of 22 patients with diaphragmatic plication through minimally invasive technique, either laparoscopic or thoracoscopic (5). On performing the plicature, the diaphragm should be rendered as taut as possible, which may be more difficult with video-assisted technique, because of the lack of full tactile feedback, and with a greater risk of damage to the abdominal viscera through a thin diaphragm (9). The incidence and intensity of post-thoracotomy pain does not seem very different to that post minimally invasive technique $(10,11)$, but further studies are necessary, on larger patient series.

The presented case is rather typical for the late diagnosis group of diaphragmatic eventration pa- tients. In such children, respiratory symptoms usually presenting as recurrent respiratory infection may be the sole clinical element pointing to the congenital anomaly. Though the problem was clearly present on physical examination and imaging data, the final diagnosis, as well as the differential diagnosis with a $\mathrm{CDH}$, was achieved during surgery, only.

\section{CONCLUSIONS}

In searching the etiology of recurrent respiratory tract infections in children, the eventration of the diaphragm is a condition easily to be missed or misdiagnosed; for that reason, it should be kept in mind. Plication of the diaphragm, by open or minimally invasive approach, is the treatment of choice, as it was also demonstrated in the presented case. Presently, the child is asymptomatic and on regular follow up. Proper informed consent was obtained from patient's parents for the publication of the article.

\section{REFERENCES}

1. J. Mouroux, N. Venissac, F. Leo, M. Alifano, F. Guillot, Surgical treatment of diaphragmatic eventration using video assisted thoracic surgery: a prospective study, Ann. Thorac. Surg. 79 (2005) 308-312.

2. Shengde Wu, Na Zang, Jin Zhu, Zhengxia Pan, Chun Wu, Congenital diaphragmatic eventration in children: 12 years' experience with 177 cases in a single institution, Journal of Pediatric Surgery (2015) : www.elsevier.com/locate/jpedsurg

3. J.M.W. Morrison, Eventration of diaphragm due to unilateral phrenic nerve paralysis, Arch. Radiol. Electrotherap. 28 (1923) 72-75

4. T.S. DeVries, B.L. Koens, A. Vos, Surgical eventration caused by phrenic nerve injury in the newborn, J. Pediatr. Surg. 33 (1998) $602-605$

5. R.K. Freeman, T.C. Wozniak, E.B. Fitzgerald, Functional and physiologic results of video-assisted thoracoscopic diaphragm plication in adult patients with unilateral diaphragm paralysis, Ann. Thorac. Surg. 81 (2006) 1853-1857

6. M.I.M. Versteegh, J. Braun, P.G. Voigt, D.B. Bosman, J. Stolk, K.F. Rabe, R.A.E. Dion, Diaphragm plication in adult patients with diaphragm paralysis leads to long-term improvement of pulmonary function and level of dyspnea, Eur. J. Cardiothorac. Surg. 32 (2007) 449-456.

7. S.M. Higgs, A. Hussain, M. Jackson, R.J. Donnelly, R.G.

Berrisford, Longterm results of diaphragmatic plication for unilateral diaphragm paralysis, Eur. J. Cardiothorac. Surg. 21 (2002) 294-297

8. D.A. Simansky, M. Paley, Y. Refaely, A. Yellin, Diaphragm plication following phrenic nerve injury: a comparison of paediatric and adult patients, Thorax 57 (2002) 613-616

9. Patel Ravisagar, Singh Abhinav, R.M. Mathur, Sisodia Anula, Eventration of diaphragm presenting as recurrent respiratory tract infections - A case report, Egyptian Journal of Chest Diseases and Tuberculosis 64 (2015), 291-293.

10. M.L. Rogers, J.P. Duffy, Surgical aspects of chronic postthoracotomy pain. A review article, Eur. J. Cardiothoracic. Surg. 18 (2000) 711-716. 11. A. Coran, N. Scott Adzick, Pediatric Surgery, vol.2, 2012, 824 